\title{
A Novel Algorithm for Human Facial Expression Recognition using Gabor Filter and Eigenvector
}

\author{
Poonam Dhankhar \\ ITM University \\ Gurgaon, India
}

\author{
Neha Sahu \\ ITM University \\ Gurgaon, India
}

\begin{abstract}
Facial Expression Recognition has always been topic of interest for research in field of human computer/machine interaction based on machine learning and image processing tools. However, the inaccuracy of such systems is always challenging. Hence to resolve this problem, a novel algorithm using Gabor Filter and Eigenvector Based Distributed Feature, is proposed in this paper. The work is enhancement of existing Face Identification by covering the challenges of Facial Expression. The approach combines face recognition along with facial expression recognition. Over the time authentication system is replaced by biometric authentication. These systems are based on some human features and behavior. The mean accuracy is improved to $93.4 \%$. The effectiveness and stability is shown in experimental results of the algorithm.
\end{abstract}

\section{Keywords}

Facial expression recognition; eigenvector; eigenvalues; Gabor filter

\section{INTRODUCTION}

The US psychologist Paul Ekman, stated that "emotions are most often confused with each other because of similarities among various facial expressions" [1]. Ekman and Friesen in their study described six emotions "happiness, sadness, anger, surprise, disgust and fear" are recognized in same manner and are the universal cultures, known as the six basic emotions of a human being [2][3]. Thus these similarities have brought difficulties with facial expression recognition reducing the accuracy. Recent years studies have proved that a learned metric can theoretically improve the performance in classification tasks [4].

Identification is one of the specific functions of biometric systems. While performing identification or detection, the system has to compares the live template with the entire set of master templates, or a subset of the dataset. This 1-to-N comparison requires establishing the identity of a person's expression with no other information other than that contained within the live template itself. The challenge with facial recognition is the facial expression [14]. The proposed algorithm will provide effective accuracy for recognition of the facial expression of an individual. In the paper, a method has been developed in which Eigenvector based facial expressions are recognized.

In the paper, a method has been presented to design an Eigenvector based facial expression recognition system. Eigenvector based features are extracted from the images. In the training phase, a set of 63 images from JAFEE database having the six basic expressions is processed. In the testing phase, the Eigenvector of an image is calculated and the corresponding Euclidean distance (EuD) of that image is computed. Euclidean distance between the Eigenvectors of that expression and the Eigenvectors of the test image is compared with the Eigenvectors of the other expressions and the one having minimum distance is classified as specific facial expression. Our method could solve the accuracy problem, and further improve the performance of facial expression recognition systems to a great level. The proposed system is designed in such a way that it first performs face recognition and once the face is recognized the particular expression is recognized. In case the face is not recognized, the expression will still be recognized providing it flexibility and making it more users friendly.

The paper is organized as- in Section 2 literature review is explained briefly. Section 3 is all about the proposed system. The experimental results are included in Section 4. Section 5 concludes the paper.

\section{LITERATURE REVIEW}

The canonical correlation analysis (CCA) to obtain features of the high resolution and low resolution images, and performed non-linear mapping favoring the nearest neighbor $(\mathrm{NN})$ classifier based on radial basis functions (RBFs). The recognition rate when tested on test images on the Facial Recognition Technology (FERET) face database [9] was $84.4 \%$, for the University of Manchester Institute of Science and Technology (UMIST) database it was 93\%, and for Olivetti Research Laboratory (ORL) database it was 95\%. The approach of Eigenface method used in most of the facial expression recognition systems was given by Turk and Pentland [11]. This method was then enhanced by Murthy and Jadon [12] and tested for the Cohn-Kanade (CK) Facial Expression database and Japanese female facial expression (JAFFE) database. The accuracy obtained fo Recently, automatic recognition of facial expression has also gained huge interest and popularity. Pentland [5] worked on the facial muscles and use dense optical flow. Their approach was intended to combine the face model, using recursive estimation. The achieved accuracy was of $98 \%$. Sebe et al. [6] experimented using different types of classifiers like k-Nearest Neighbor (kNN), Support Vector Machines (SVMs), and Bayesian Networks and decision tree based classifiers and developed an Authentic Facial Expression Analysis. Peter W. McOwan and Keith Anderson [7] used an enhanced ratio template algorithm to recognize the front view of the face in moving face. He used support vector machine as a classifier (SVM) and computed the recognition rate of $81.82 \%$.

The recognition accuracy achieved for the Gabor-based elastic graph matching technique was $90.5 \%$ whereas for the normalized morphological based elastic graph matching technique was $91.8 \%$. 
Jun OuXiao-Bo Bai Yun Pei, Liang Ma, Wei Liu [8] presented a system that used 28 facial feature key-points in images detection and Gabor wavelet filter provided with five different frequencies and eight orientations at different angles. On the basis of actual demand, it was capable to extract the feature of low quality facial feature image target, having powerful automatic facial expression recognition. The results shows that the performance of the method thus developed had excellent recognition rates, when applied to facial expression recognition system fair rate of accuracy were achieved.

In Year 2011, S Prasad performed a work," Real-time Face Recognition System with Dynamic Training and Enhanced Multi-Algorithm Face Recognition". Main objective is to realize a smart, maintenance-free system with effective design; Presented research develops an immaculate realtime face recognition system with dynamic training and improved multi-algorithm face recognition.

Huang and He [9] proposed a method to improve the face recognition of low resolution images. They performance CK was efficient but when tested on JAFEE the accuracy was not reasonable.

Mandeep Kaur, Rajeev Vashisht and Jalandhar Nirvair Neeru in year 2010 [14] proposed a system based on Principal Component Analysis and Singular Value Decomposition. The experiment was tested on JAFEE and accuracy was of each expression was calculated separately. Six basic expressions namely happy disgust surprise angry and sad were seen to have 95.00, 70.00, 85.00, 60.00 and 90.00 recognition rate using PCA was achieved.

In 2012 Bin Jiang, Ke-bin Jia, Qiang Wu [13], presented a novel algorithm for facial expression recognition based on discriminative component analysis (DCA) The algorithm when tested on JAFEE the observed rate of accuracy was $82.42 \%$. In our proposed work we aim to aim to improve the accuracy and enhance the performance of human facial expression recognition system for JAFEE database using eigenvector and Gabor filter.

\section{PROPOSED SYSTEM}

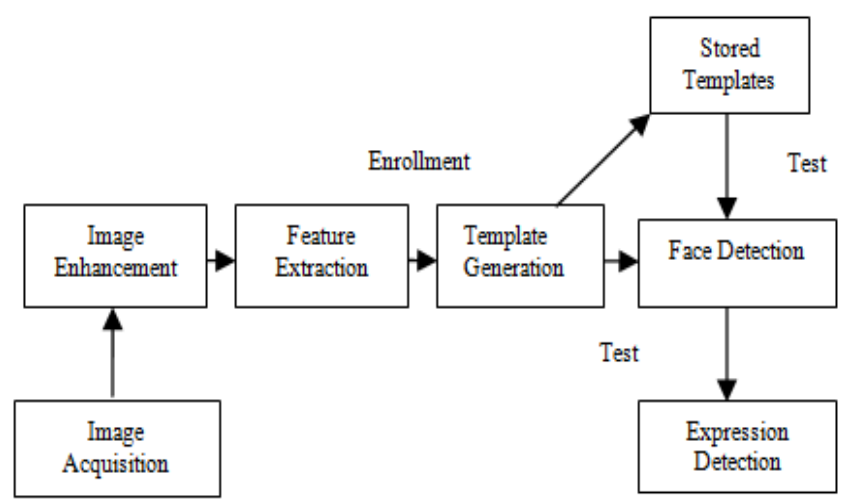

Figure 1: Block diagram of Facial Expression Recognition Systems

\subsection{Image acquisition and enhancement}

Images are taken from JAFEE database. 21samples of six basic expressions of three females are taken i.e. total 63 are included in the training dataset. The images which are in rgb will fit be converted to grayscale. Images are resized to a common size i.e. [200,200].
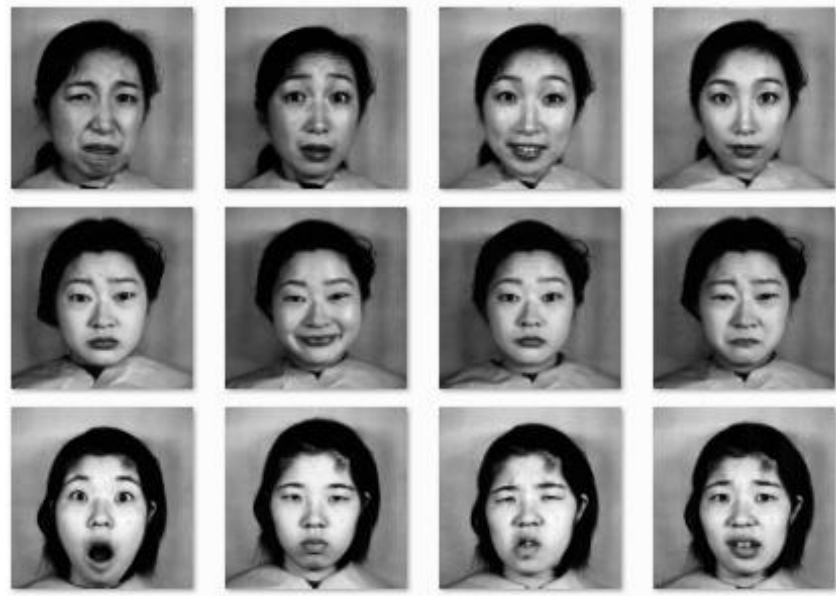

Figure 2: Samples in JAFEE

Firstly the images are pre-processed before going to further steps.

\subsection{Feature Extraction}

Gabor Filter is used for feature extraction. Gabor being band pass filters are most commonly used in image processing for feature extraction. In our system the Gabor filters are combined with Eigenvectors to enhance the performance and accuracy.
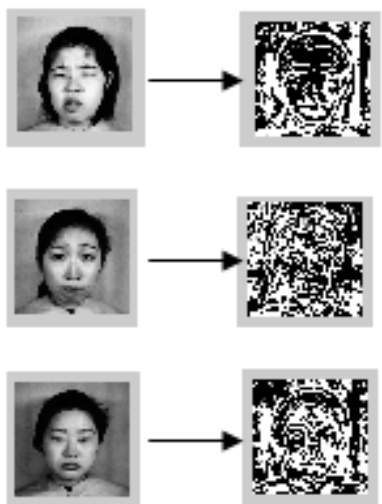

Figure 3: Feature extraction

\subsection{Template Generation}

The work classifies the six basic universal emotions after the face is recognized and training of images completes. Eigenvectors and Eigenvalues of different segments of the image is calculated and stored. For a single expression type, say happy, the selection of a particular feature and the corresponding matrix obtained is stored in a variable of dimension $\mathrm{R} \times \mathrm{S}$. The same procedure is followed for rest of the images based on the expression. Eigenvectors and Eigenvalues are calculated and stored in a matrix.

Difference between the mean of the image the mean centered feature image, the vectors are obtained. These image vectors are stored in matrix form only. After finding the covariance matrix of each image, eigenvectors and Eigenvalues are generated.

The classification of the expression takes place using EuD based decision making method, thus the closest one is declared as the result i.e. the particular expression. 


\subsection{Classification}

The classification of the expression is based on the Euclidean distance. By computing the distance between the images in the database and one which is being tested

At the time of testing, the Euclidean distance (EuD) is calculated between the testing (input) image Eigenvector and the Eigen subspaces of every expression, and the one having minimum EuD is recognized as the particular expression of that input image. The same process is followed for face recognition. The equation for Euclidean distance is given by

$$
\mathrm{EuD}=\sqrt{\sum\left(X_{2}-X\right)^{2}}
$$

\section{EXPERIMENTAL RESULTS AND DISCUSSION}

The simulation is performed on MATLAB R2013b. the experiment is done on Japanese Female Expression database (JAFEE [15]). It is one of the most common databases used by researchers across the world. We have chosen 21 samples of the expressions of three different persons. in order to eliminate the noise, image enhancement is performed at the time of image pre-processing.

Figure 4, shows the actual GUI.

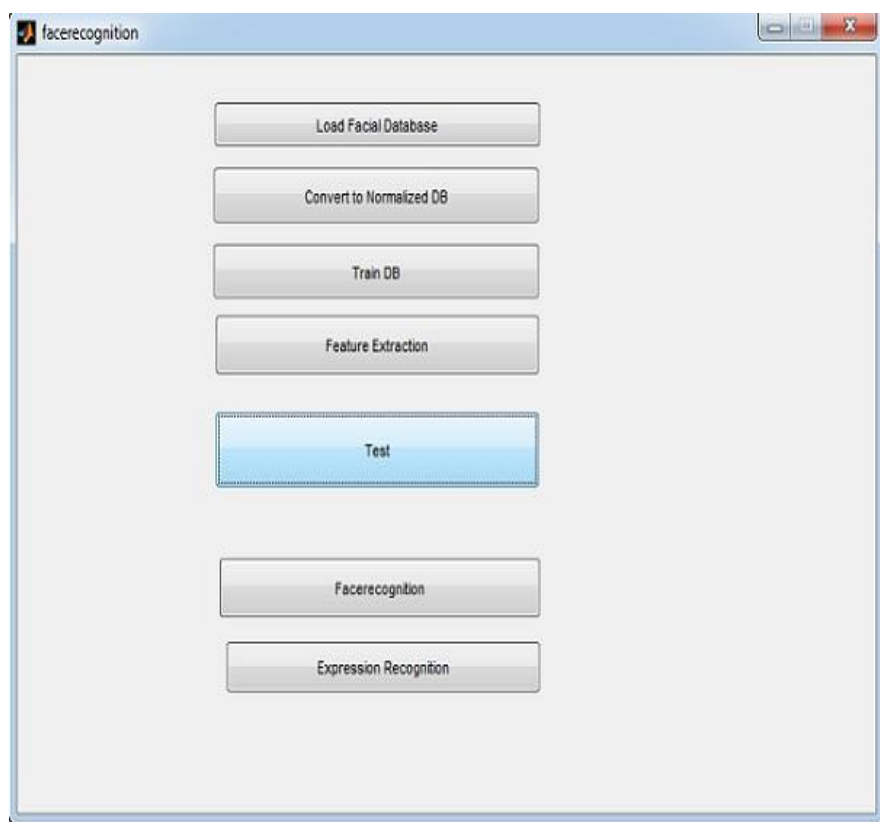

Figure 4: GUI

Images shown in Figure 5 and 6 were tested and results are shown.

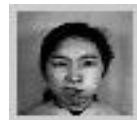

Tested (input) image 1

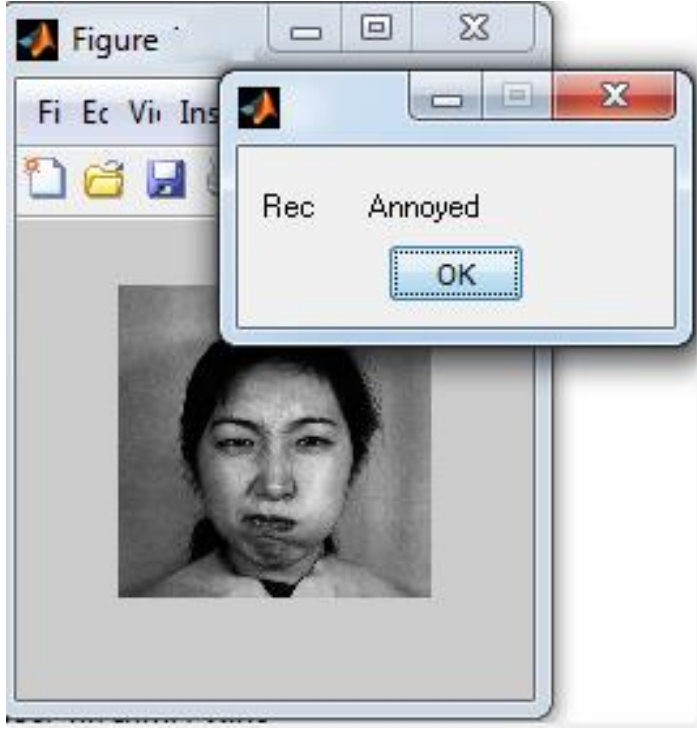

Figure 5: Annoyed expression

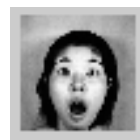

Tested (input) image 2

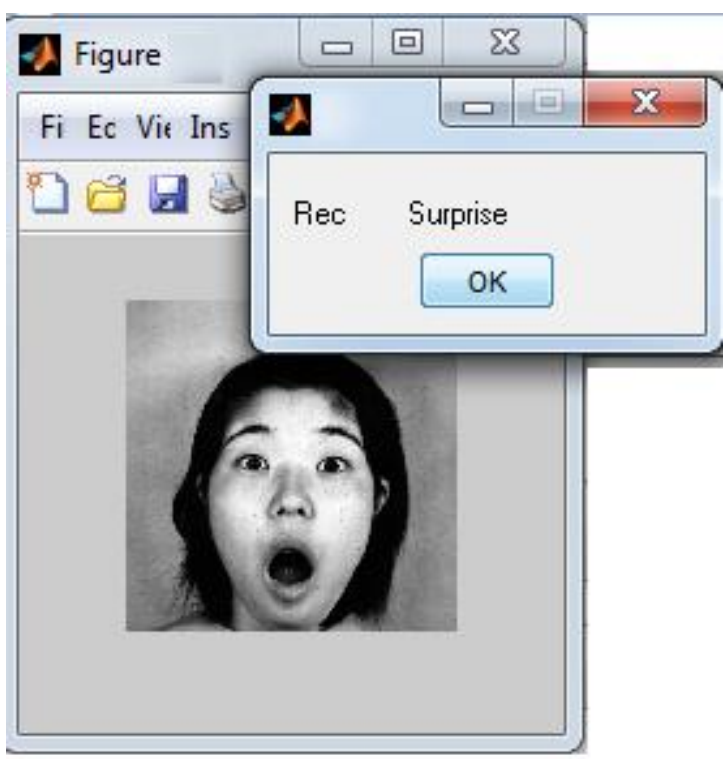

Figure 6: Surprise Expression

In figure 7 , the accuracy rate on different size of dataset is shown. The accuracy for dataset size 10, 20. 30, 40 and 50 was computed to be $90,92.34,94.23,94.33$ and, 95.22 respectively. 


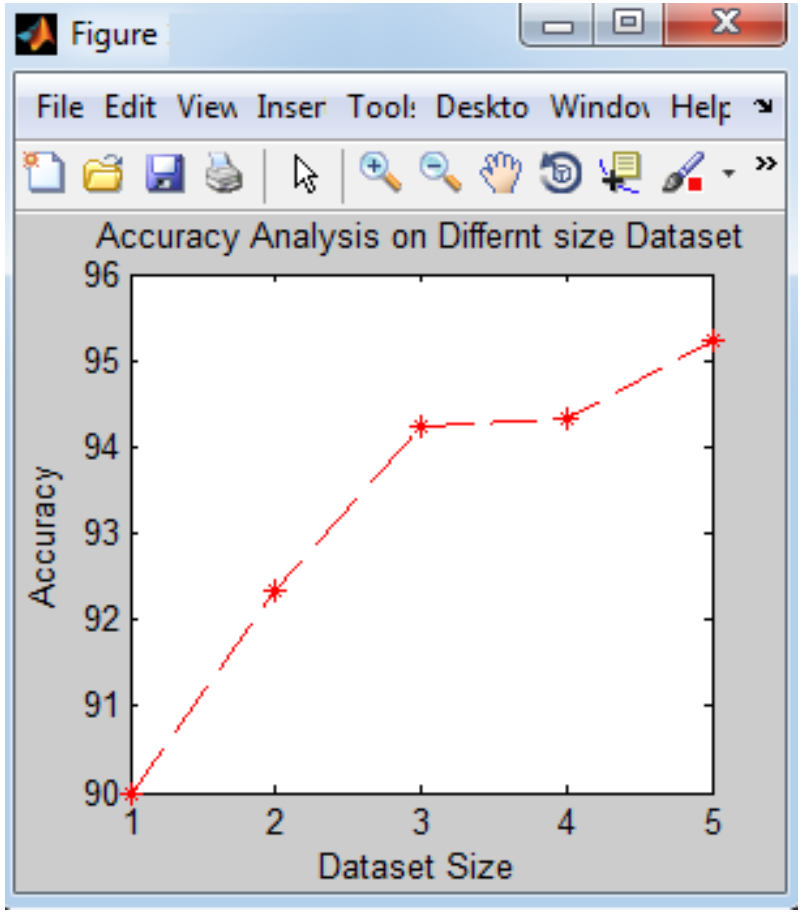

Figure 7: Accuracy analysis

The average accuracy thus recorded is " $93.15 \%$ ".

In figure 8 , comparison is shown between the existing system and the proposed system. It is clear that the proposed system has a better accuracy rate.

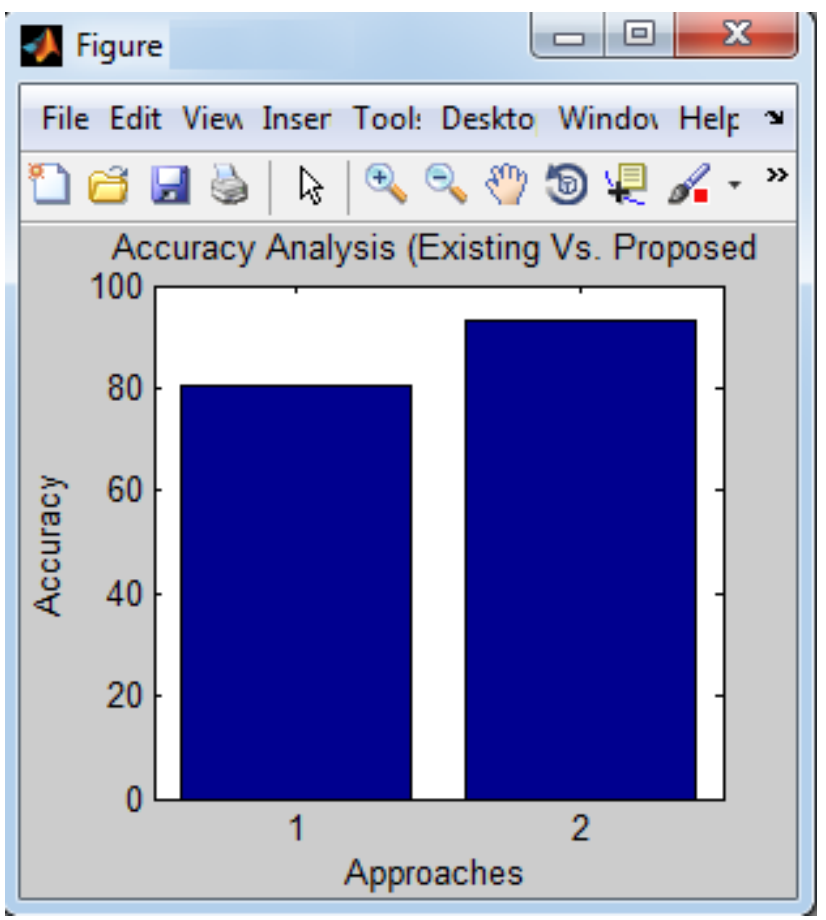

Figure 8: Comparison graph

\section{CONCLUSION}

The experimental results show the efficacy of our proposed method, primarily used for recognition of the face following the six basic expressions. The recognition rate obtained for the proposed system is $93.15 \%$.
In humans expressions are most common part for communication and humans can recognize the facial expressions effortlessly and far more accurately than any system or machine without any delay. This is still a challenging task. Our future work is developing a system with such accuracy and including real time implementation.

\section{REFERENCES}

[1] P. Ekman, "FACS vs. F.A.C.E", Paul Ekman Group, 2009.

[2] P. Ekman, E. R. Sorenson, and W. V. Friesen, "Pan cultural elements in Facial displays of emotion" Science, New Series, vol. 164, no. 3875, pp. 86-88, April 4,1969

[3] P. Ekman and W.V. Friesen, "Manual for the Facial Action Coding System," Consulting Psychologists Press, 1977. "A Boosting Framework for Visuality-Preserving Distance Metric Learning \& its Application to Medical Image Retrieval", IEEE Transaction on Pattern Analysis and Machine Intelligence, vol.32, no.1, pp.33-44.

[4] K. Mase and A. Pentland, "Recognition of facial expression from optical flow," IEICE Trans. E, vol. 74, pp. 408-410, 1991.

[5] Cohn, J.F., Kanade, T., Lien, J.J., 1998,"Automated Facial Expression Recognition Based on FACS Action Units", Proc. Third IEEE Int. Conf. Automatic Face and Gesture Recognition, pp. 390-395.

[6] Kapoor, A., Picard, R. W., Yuan Qi. , 2003, "Fully Automatic Upper Facial Action Recognition", IEEE International Workshop on Analysis and Modeling of Face and Gestures, pp.195.

[7] Yang Zhang," Local Binary Patterns for Face Recognition Under Varying Variations", CSIIRW '10, April 21-23, Oak Ridge, Tennessee, USA ACM 978-14503-0017-9.

[8] H. Huang and H. He, "Super-Resolution Method for Face Recognition Using Nonlinear Mappings on Coherent Features", IEEE Transactions on Neural Networks, vol. 22, no. 1, pp. 121-130, January 2011.

[9] M. Turk and A. Pentland, "Eigenfaces for recognition," Journal of Cognitive Neuroscience, vol.13, no. 1, pp. 7186, 1991.

[10] G. R. S. Murthy and R. S. Jadon, "Effectiveness of Eigenspaces for Facial Expressions Recognition" International Journal of Computer Theory and Engineering, vol. 1, no. 5, pp. 638-642, December 2009.

[11] R. Zhi, M. Flierl, Q. Ruan, and W. B. Kleijn, "GraphPreserving Sparse Nonnegative Matrix Factorization with Application to Facial Expression Recognition”, IEEE Transactions on Systems, Man and Cybernetics-Part B: Cybernetics, vol.41, no. 1 pp. 38-52, February 2011.

[12] Bin Jian, Ke-bin Jia and Qiang Wu, "A Novel Algorithm of Facial Expression Recognition Based on DCA", IEEE International Conference on Intelligent Information Hiding and Multimedia Technology”, 2012.

[13] Jeemoni Kalita and Karen Das, "Recognition of Facial Expression Using Eigenvector Based Distributed Features and Euclidean Distance Based Decision Making Technique", IJACSA) International Journal of Advanced Computer Science and Applications, Vol. 4, No. 2, 2013. 
[14] L. Zhang and D. Tjondronegoro, "Facial Expression Recognition Using Facial Movement Features", IEEE Transactions on Affective Computing, vol. 2, no. 4, pp. 219-229, October-December 2011.

[15] Cirelo, M., Cohen, I., Cozman, F., Huang, T., Sebe, N.

[16] 2004, "Semi-supervised learning of classifier", Theory, algorithms, and applications to human-computer interaction. Vol.26, No. 12, pp.1553-1567.

[17] S Prasad," Real-time Face Recognition System with Dynamic Training and Enhanced Multi-Algorithm Face Recognition", International Conference and Workshop on Emerging Trends in Technology (ICWET 2011) TCET, Mumbai, India ACM 978-1-4503-0449-8/11/02.

[18] Nadia AL-Hassan," Enhancing Face Recognition at a Distance using Super Resolution", MM\&Sec'12, September 6-7, 2012, Coventry, United Kingdom. ACM 978-1-4503-1418-3/12/09.

[19] S. Wang, Z. Liu, S. Lv, Y. Lv, G. Wu, P. Peng, F. Chen, and X. Wang, "A Natural Visible and Infrared Facial Expression Database for Expression Recognition and Emotion Inference", IEEE Transactions on Multimedia, vol. 12, no.7, pp. 682-691, November 2010.

[20] H. A. Elfenbein, A. A. Marsh, and N. Ambady, "Emotional Intelligence and the Recognition of Emotion from Facial Expressions" in The Wisdom of Feelings: Processes Underlying Emotional Intelligence

[21] Yong Ma," Person-specific Age Estimation under Ranking Framework", ICMR '11, April 17-20, Trento, Italy ACM 978-1-4503-0336-1/11/04

[22] H B Kekre," Transform Based Face Recognition with Partial and Full Feature Vector using DCT and Walsh Transform", International Conference and Workshop on
Emerging Trends in Technology (ICWET 2011) TCET, Mumbai, India ICWET'11, February 25-26, 2011, Mumbai, Maharashtra, India. ACM 978-1-4503$0449-8 / 11 / 02$

[23] Zhongkai Han," A Discriminated Correlation Classifier for Face Recognition", SAC'10 March 22-26, 2010, Sierre, Switzerland. ACM 978-1-60558-638-0/10/03

[24] Per Kvarnbrink," Biometric Interaction - A Case Study of Visual Feedback and Privacy Issues in New Face Recognition Solutions", CHI 2013 Extended Abstracts, April 27-May 2, 2013, Paris, France. ACM 978-1-4503$1952-2 / 13 / 04$

[25] K. Anderson and P. W. McOwan, "A Real-Time Automated System for the Recognition of Human Facial Expressions" IEEE Transactions on Systems, Man and Cybernetics-Part B: Cybernetics, vol. 36, no. 1, pp. 96105, February 2006.

[26] Vikas Maheshkar," DCT-Based Unique Faces for Face Recognition using Mahalanobis Distance", IITM'10, December 28-30, 2010, Allahabad, UP, India. ACM 978-1-4503-0408-5/10/12.

[27] Seiji TAKEDA," Implicit Context Awareness by Face Recognition", MoMM2011, 5-7 December, 2011, Ho Chi Minh City, Vietnam.ACM 978-1-4503-0785-7/11/12

[28] Divya R.," Astroid Shaped DCT Feature Extraction For Enhanced Face Recognition", CUBE 2012, September 3 to 5, 2012, Pune, Maharashtra, India. ACM 978-1-4503$1185-4 / 12 / 09$

[29] Seiji TAKEDA," Implicit Context Awareness by Face Recognition", MoMM2011, 5-7 December, 2011, Ho Chi Minh City, Vietnam.ACM 978-1-4503-0785-7/11/12 